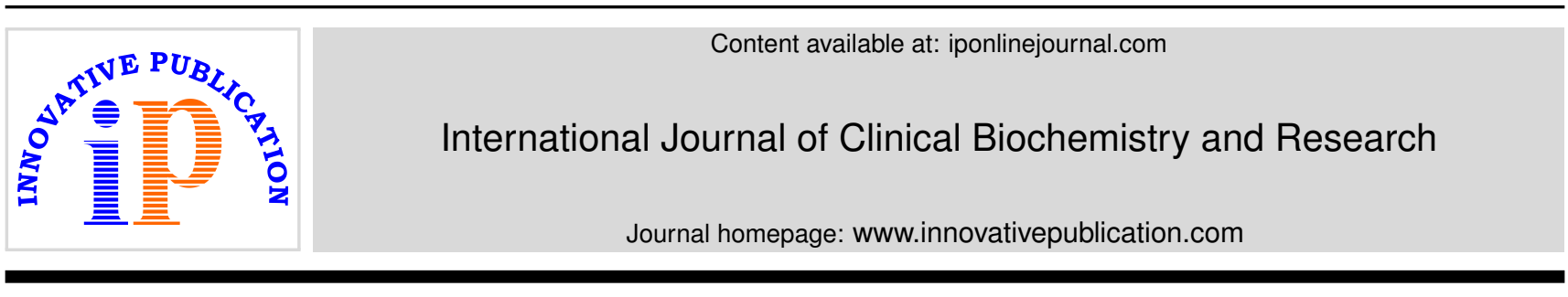

Original Research Article

\title{
A study of serum thyroid hormones in organophosphorus compounds poisoning patients
}

\author{
Ranjith Kumar $\mathbf{C}^{1, *}$ \\ ${ }^{1}$ Dept. of Biochemistry, S.V.S. Medical College, Mahabubnagar, Telangana, India
}

\section{A R T I C L E I N F O}

\section{Article history:}

Received 12-02-2020

Accepted 25-03-2020

Available online 30-06-2020

\section{Keywords:}

Organophosphorus compounds (OPC)

Total triiodothyronine(TT3)

Total tetraiodothyronine(TT4)

Thyroid stimulating hormone (TSH)

Cholinesterase enzyme

\begin{abstract}
A B S T R A C T
Background: Individuals have the chance to get exposed to organophosphates either at domestic, or industrial spheres. Organophosphate poisoning is a worldwide problem. Organophosphate insecticides inhibit Cholinesterase enzymatic activity. Chlorpyrifos, Phorate 10\% granules and Monocrotophos are insecticides and pesticides commonly used in India by farmers.

Aim: The present study aims at determining the effect oforganophosphate compounds on thyroid profile in individuals exposed to acute poisoning.

Materials and Methods: In this study, those individuals who were acutely presented at the emergency department, SVS Medical College, Mahabubnagar, with a history of intentional or accidental intake of either chlorpyrifos or Phorate $10 \%$ granules or Monocrotophos (Organophosphorus compounds) were taken as cases. Intake of these compounds was confirmed by analyzing serum cholinesterase levels. Those patients having a serum cholinesterase levels value below $4000 \mathrm{U} / \mathrm{L}$ were included in this study. Simultaneously, the sample was analyzed for thyroid profile that includes Total triiodothyronine (TT3), Total tetraiodothyronine (TT4) and Thyroid stimulating hormone (TSH). Reanalyzing of thyroid profile was done after a time gap of one month from the day of discharge from hospital after recovery.

Results: Total T3, Total T4 and TSH values showed statistically significant change $(\mathrm{p}$-value $<0.005)$ between the acute poisoning phase and one month follow up phase of after recovery.

Conclusion: Total T4 values are significantly higher in acute organophosphorus poisoned phase mimicking thyrotoxicosis, and return to normal biological reference range after recovery, whereas TSH values in acute opiates poisoned phase were significant lower than that of recovery phase.
\end{abstract}

(C) 2020 Published by Innovative Publication. This is an open access article under the CC BY-NC license (https://creativecommons.org/licenses/by-nc/4.0/)

\section{Introduction}

There are two forms of cholinesterase; acetyl cholinesterase and cholinesterase or also commonly referred to as psuedocholinesterase. Acetyl cholinesterase is found predominantly in erythrocytes. Cholinesterase is synthesized in liver and is present in plasma and is the form of the enzyme routinely measured. Cholinesterase is most commonly measured as an indicator of exposure to anticholinesterases (organophosphates, including many insecticides). cholinesterase Organophosphorus (OP) compounds are used as herbicides and pesticides. Incidence

\footnotetext{
* Corresponding author.

E-mail address: write2drranjith@gmail.com (R. Kumar C).
}

of Organophosphate poisoning is estimated to cause 0.25 0.35 million deaths per year globally. ${ }^{1,2}$ Organophosphate poisoning is one of the major public health problems in developing countries, resulting in significant morbidity and mortality. ${ }^{3}$ Most of the OP poisoning and subsequent deaths occur in countries like India, following a deliberate self ingestion, particularly in adult group as highly toxic pesticides are readily available at the moment of stress due to family problems, failure in relation and exam phobia etc. Occupational exposure is also observed in adults due to unprotected use. Accidental poisoning occurs in children due to their innovative and exploratory nature. Many studies have shown that accidental poisoning has lower mortality than deliberate self poisoning. Few 
studies have been done in India on organophosphate poisoning but none of them measured the thyroid status during acute poisoning. This study tends to observe the effects of most commonly available organophosphorus compounds like Chlorpyrifos, Phorate $10 \%$ granules and Monocrotophos on thyroid hormones. As per WHO recommended classification of pesticides by hazard and guidelines to classification 2009, Phorate granules related to Ia (extremely hazardous), Monocrotophos related Ib (highly hazardous) and Chlorpyrifos is a group member of class II (moderately hazardous).

\section{Materials and Methods}

This prospective study was performed in Medical Intensive Care Unit and Clinical biochemistry department of S.V.S Medical College \& Hospital. Subjects were selected based on the following inclusion and exclusion criteria.

\subsection{Inclusion criteria}

1. Serum cholinesterase value below $4000 \mathrm{U} / \mathrm{L}$ at admission

2. Proof of organophosphorus poison..

\subsection{Exclusion criteria}

1. Nonspecific history and no proof of organophosphorus poisoning.

2. Hypothyroid and Hyperthyroid patients.

3. Individuals on medication for hypothyroid and hyperthyroid conditions.

4. Death during treatment.

5. Cholinesterase value ranges between $4000-12000$ $\mathrm{U} / \mathrm{L}$ at admission.

Thirty patients with organophosphate poisoning were included in this study. Among them eighteen were males and twelve were females aged $25.9 \pm 10.99$ yrs. Consent from patient's relatives was taken after explaining the academic purpose of this study. The study has not recorded the social status, educational qualifications, marital status and cause of OPC intake.

$2 \mathrm{ml}$ of blood sample was collected under aseptic conditions in a clot activator tube. Butyrylthiocholine method is used for analyzing serum cholinesterase enzyme levels in Beckman AU480 auto analyzer. The samples were processed within 4 hours of collection so as to maintain cholinesterase half life. The hemolyzed samples were rejected as RBC's cholinesterase would have leaked into the serum. The same sample was used for thyroid profile estimation which includes total triiodothyronine (TT3), total tetra iodothyronine (TT4) and thyroid stimulating hormone (TSH). Chemiluminescence method was used for analyzing thyroid profile in Beckman Access 2 immunoassay analyzer.
After recovery from the acute episode of poisoning, the follow up samples were taken after one month of discharge and were reanalyzed for the serum TT3, TT4 \&TSH, and these values were compared with their previous values using t- test. This study was conducted from August2018 to January 2019. The study was approved by Institutional Ethical Committee.

\section{Results}

The total T3 values on both occasions, were found to be within biological reference range, but the recovery phase values were higher than exposure period values, and this difference was statistically significant. Total $\mathrm{T} 4$ values during the acute exposure were higher than the biological reference range, mimicking thyrotoxicosis, and then dropped down to biological reference limits after recovery. Elevated levels of TT4 during acute phase of poisoning were statistically significant. TSH values at exposure were lower than that of recovery phase, however statistically significant elevation was observed in the recovery phase. Thus in acute phase of organophosphorus poisoning, thyrotoxicosis was observed and patients returned to euthyroid state during recovery phase.

\section{Discussion}

So far epidemiological, clinical symptoms and treatment studies have been done on the back ground of organophosphorus poisoning. ${ }^{4,5}$ There is large scope for the studies to reveal the actual endocrine glands response to massive organophosphorus chemical exposures like in poisoning cases. Men are more in number $60 \%(\mathrm{n}=18)$ than women $40 \%(n=12)$. In India males are main working group, hence prone for extensive stress and unhealthy routine which is the main reason for increasing number of cases day by day. This study has shown similar results of previous studies in regard to gender exposure. ${ }^{6}$ Exposure is less in children than in adults. (only 2 children and 28 adults were included in this study).

Two Indian ${ }^{5,7}$ studies and one Sri Lankan ${ }^{8}$ study showed that majority of the cases were admitted due to ingestion of WHO class Ib pesticides. Monocrotophos contributed major portion among them. ${ }^{7}$ Some studies mainly describe the signs and symptoms, ${ }^{9}$ but there are no studies that describe effects of organophosphorus compound on the thyroid gland before treatment and after recovery from organophosphorus poisoning. No follow up studies have been done and there is no documentation on chronic complications. Finally, based on the observations of this study results, it was concluded that organophosphorus chemicals like Chlorpyrifos, Phorate $10 \%$ granules and Monocrotophos in toxic doses might act on the thyroid follicles of thyroid gland and release $\mathrm{T} 4$ from stores. There will be one more possibility is that these opiates might prevents peripheral conversion of TT4 
Table 1:

\begin{tabular}{lcccccc}
\hline & \multicolumn{2}{c}{ TT3 } & \multicolumn{2}{c}{ TT4 } & \multicolumn{2}{c}{ TSH } \\
Mean & At exposure & After recovery & At exposure & After recovery & At exposure & After recovery \\
SD & 0.904 & 1.152 & 12.32 & 7.69 & 1.448 & 2.366 \\
p- value & 0.43 & 0.29 & 2.733 & 1.108 & 0.8068 & 0.9839 \\
\hline
\end{tabular}

to TT3 by inhibiting the deiodinase enzymes at liver and kidneys, which might cause thyrotoxicosis during acute OP poisoning. This observation confirmed by significant difference between TT3 values of both phases.

Most of the studies ${ }^{10-13}$ concluded their findings by studying the occupational exposure groups like farmers and factory workers. They established different relations between organophosphates and thyroid hormones. A Pakistan based study ${ }^{10}$ established a relation between the pesticides and endocrine dysfunction in cotton crop agricultural workers. In their case control study, they found a significant decreased serum FT4 levels in cotton pickers case group against control group. They found thyroid disturbances in pesticide exposure cases. Some studies established no effect on serum levels of TSH and FT4 in occupational exposure farm workers to organophosphates and carbamate pesticides. ${ }^{11}$ Other studies at investigating the effect of agropesticides on male farmers serum triiodothyronine (T3) and thyroxine (T4) levels in Djutitsa. Compared to the control group, there was no significant difference in thyroid hormones (T3 and T4) levels in cases group. ${ }^{12}$

One study reveal their findings based on fipronil manufacturer industry workers. They established non significant negative correlation between serum TSH and fipronil concentration, raising the possibility that fipronil has a central inhibitory effect on TSH secretion in humans. 13

Based on the above findings we can summarize the effects of pesticides on thyroid hormones levels in serum. The pesticides modifying the hormone levels by acting at different sites, those are either at pituitary- thyroid axis or thyroid gland level or peripheral conversion by deiodinase enzyme level. They effects depend not only on the exposure pesticides concentration but also on exposure period to pesticides.

We can divide the risk to exposure pesticides people into 3 groups. First group is farmers, those are at less level of concentration exposure and lesser time than the remaining two groups. In this group most of the case control studies ${ }^{11,12}$ withdrawn the non significant changes of thyroid hormones. Second group is industrial workers, these are at high level concentration and longer time exposure to pesticides. They have chances of multi route exposure than other groups. Only few studies taken place on thyroid effects by pesticides in this group. They establish decreased TSH levels against pesticide concentration. Third group is intentional or accidental exposed persons, they have chances of expose to medium level concentration when compare to first and second group. The exposure time is based on the gap between the exposure and medical treatment placement. There is no such studies haven't taken place about organophosphates effects on thyroid hormones in this group, but this study try to explain the same.

\section{Conclusion}

This study concluded the observation that in acute organophosphorus compounds poisoning, patients show thyrotoxicosis picture, because of opiates activity on thyroid gland stores and releases the TT4 as well as prevent the peripheral conversion of TT4 to TT3 by inhibiting the deiodinases. After treatment for the acute stage, these patients retained the euthyroid state. The effects of organophosphates on serum thyroid hormones levels depend on the concentration and duration of exposure to pesticides.

\section{Limitations of this study}

The results are based on single study center data evaluation. There is no documentation of evaluation of endocrine glands other than that of thyroid.

\section{Acknowledgements}

The author thank S.V.S Medical College, Telangana for their essential support.

\section{Sources of Funding}

Nil.

\section{Conflict of Interest}

None.

\section{References}

1. Peshin SS, Srivastava A, Halder N, Gupta YK. Pesticide poisoning trend analysis of 13 years: A retrospective study based on telephone calls at the National Poisons Information Centre, All India Institute of Medical Sciences, New Delhi. J Forensic Leg Med. 2014;22:57-61.

2. Kır MZ, Öztürk G, Gürler M, Karaarslan B, Erden G, Karapirli M. Pesticide poisoning cases in Ankara and nearby cities in Turkey: An 11-year retrospective analysis. J Forensic Leg Med. 2013;20(4):274-7.

3. Buckley NA, Roberts D, Eddleston M. Overcoming apathy in research on organophosphate poisoning. BMJ. 2004;329:1231-3.

4. Mishra A. Epidemiological Study of Medicolegal Organophosphorus Poisoning in Central Region of Nepal. J Forensic Res. 2012;03(09). 
dol:10.4T/2/215/-7145.1000167.

5. Thunga G, Sam KG, Khera K. Sureshwar Pandey and Sudha Vidya Sagar. Evaluation of incidence, clinical characteristics and management in organophosphorus poisoning patients in a tertiary care hospital. J Toxicol Environ Health Sci. 2010;2(5):73-6.

6. Gupta SK, Kumar S, Sheikh MI. Study of Organophosphorus Poisoning in Surat, India. JIAFM. 2006;28(3).

7. Rao CS, Venkateswarlu V, Surender T, Eddleston M, Buckley NA. Pesticide poisoning in south India: opportunities for prevention and improved medical management. Trop Med Int Health. 2005;10(6):581-8

8. Hoek W, Flemming K. Analysis of 8000 Hospital Admissions for Acute Poisoning in a Rural Area of Sri Lanka. Clin Toxicol. 2006;44:225-31.

9. Chintale K, Patne S, Chavan S. Clinical profile of organophosphorus poisoning patients at rural tertiary health care centre. Int J Adv Med. 2016;3(2):268-74.

10. Khan DA, Ahad K, Ansari WM, Khan H. Pesticide Exposure and Endocrine Dysfunction in the Cotton Crop Agricultural Workers of Southern Punjab, Pakistan. Asian Pac J Public Health. 2013;25:18191.
11. Contreras LM, Perez RG, Rojas G, I C. Occupational exposure to organophosphate and caramate pesticides affects sperm chromatin integrity and reproductive hormone levels among Venezuelan farm workers. J Occup Health. 2013;55(3):195-203.

12. Manfo FPT, Moundipa PF, Déchaud H, Tchana AN, Nantia EA, Zabo MT, et al. Effect of agropesticides use on male reproductive function: A study on farmers in Djutitsa (Cameroon). Environ Toxicol. 2012;27:423-32.

13. Herin F, Boutet-Robinet E, Levant A, Dulaurent S, Manika M, Galatry-Bouju F, et al. Thyroid Function Tests in Persons with Occupational Exposure to Fipronil. Thyroid. 2011;21:701-6.

\section{Author biography}

Ranjith Kumar C Associate Professor

Cite this article: Kumar C R. A study of serum thyroid hormones in organophosphorus compounds poisoning patients. Int J Clin Biochem Res 2020;7(2):272-275. 